\title{
Personalized medicine (PM)
}

Sebastian Schleidgen, Corinna Klingler, Teresa Bertram, Wolf H Rogowski, Georg Marckmann

\section{Source}

Sebastian Schleidgen, Corinna Klingler, Teresa Bertram, Wolf H Rogowski, Georg Marckmann. (2013). What is personalized medicine: sharpening a vague term based on a systematic literature review. BMC Med Ethics, vol. 14 (1). doi:10.1186/1472-6939-14-55.

PM seeks to improve stratification and timing of health care by utilizing biological information and biomarkers on the level of molecular disease pathways, genetics, proteomics as well as metabolomics. 